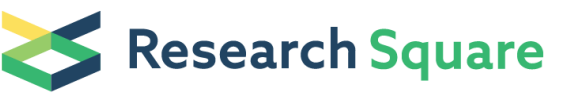 \\ Preprints are preliminary reports that have not undergone peer review. \\ They should not be considered conclusive, used to inform clinical practice, or referenced by the media as validated information.
}

\section{Proportion of Neutrophils in the White Blood Cells as a Useful Marker for Predicting Bacteremic Acute Cholangitis}

Atsushi Yamaguchi ( $\sim$ yamaguchiatsushi@mail.hosp.go.jp )

National Hospital Organization Kure Medical Center Chugoku Cancer Center

Kaoru Wada

National Hospital Organization Kure Medical Center Chugoku Cancer Center

Riho Moriuchi

National Hospital Organization Kure Medical Center Chugoku Cancer Center

Kanae Tao

National Hospital Organization Kure Medical Center Chugoku Cancer Center

Hirona Konishi

National Hospital Organization Kure Medical Center Chugoku Cancer Center

Yuzuru Tamaru

National Hospital Organization Kure Medical Center Chugoku Cancer Center

Ryusaku Kusunoki

National Hospital Organization Kure Medical Center Chugoku Cancer Center

Toshio Kuwai

National Hospital Organization Kure Medical Center Chugoku Cancer Center

Hirotaka Kouno

National Hospital Organization Kure Medical Center Chugoku Cancer Center

Hiroshi Kohno

National Hospital Organization Kure Medical Center Chugoku Cancer Center

\section{Research Article}

Keywords: Acute cholangitis, Bacteremia, Neutrophil, Neutrophil proportion in white blood cells

Posted Date: July 14th, 2021

DOl: https://doi.org/10.21203/rs.3.rs-532812/v1

License: (ㄷ) This work is licensed under a Creative Commons Attribution 4.0 International License. Read Full License 


\section{Abstract \\ Background}

A positive hemoculture in acute cholangitis is serious, and accordingly, a predictor for bacteremia at initial diagnosis would be particularly useful. We investigated the association between neutrophil proportion in the white blood cell count (Neut\%) and bacteremic acute cholangitis.

\section{Methods}

In 166 patients of acute cholangitis diagnosed with Tokyo guideline 2013 from April 2014 to March 2015, ninety-four patients underwent blood culture assessment and were divided into a positive hemoculture $(n=48)$ and negative hemoculture $(n=46)$ group and these two group were compared. A receiver operating characteristic curve analysis was used to evaluate the predictive ability of Neut\% and other inflammatory markers.

\section{Results}

Neut $\%$ was significantly higher in the positive hemoculture $(91.7 \pm 4.0 \%$ vs. $82.5 \pm 9.0 \%, P<0.0001)$. A cutoff Neut $\%$ value of $89.7 \%$ was strongly associated with bacteremia (area under the curve 0.86 , sensitivity $77 \%$, specificity $80 \%$ ). A Neut $\%$ of $\geq 89.7 \%$ was a predictor of positive hemoculture in the univariate $(P<0.0001)$ and multivariate analyses $(P<0.001)$. Patients with a Neut $\% \geq 89.7 \%$ needed early biliary drainage $(30 / 46,65.2 \%$ vs. $18 / 48,37.5 \%, P=0.0063)$.

\section{Conclusions}

Neut\% is an independent predictor of bacteremia and prognosis in patients with acute cholangitis and may contribute to decision-making of early biliary drainage.

\section{Background}

Acute cholangitis can be fatal and requires biliary decompression at an appropriate time for recovery [1]. A positive hemoculture is a predictive factor for prognosis of acute cholangitis [2-4]. Unfortunately, a blood culture result cannot be instantly obtained upon admission and cannot be used to assess the severity of disease or to help in the decisionmaking process for urgent or early biliary drainage. Therefore, an excellent predictor of bacteremia would be useful for patients with acute cholangitis. Some biomarkers have been reported to predict prognosis and bacteremia in patients with acute cholangitis; for example, serum procalcitonin (PCT) levels [6-8]. Although, to the best of our knowledge, a credible predictive biomarker for bacteremia in patients with cholangitis has not yet been confirmed.

In our current practice, we have noticed that the neutrophil proportion in the white blood cell count (Neut\%) is frequently higher in patients with a positive hemoculture than those with a negative hemoculture in acute cholangitis. Accordingly, we retrospectively investigated the association between Neut\% and bacteremia and prognosis in patients with acute cholangitis.

\section{Methods}

This retrospective study included 166 patients diagnosed with acute cholangitis according to the Updated Tokyo Guidelines for acute cholangitis and acute cholecystitis 2013 (TG13) [9] between April 2014 and March 2015 at the 
National Hospital Organization Kure Medical Center and Chugoku Cancer Center. The number of patients who underwent hemoculture without pre-administration of antibiotics at the time of the first medical examination was 115 . Of these patients with acute cholangitis, we analyzed 94 patients who did not have any factors affecting white blood cell (WBC) count, serum C-reactive protein (CRP) level, body temperature, and Neut\% (Fig. 1). This study was performed in accordance with the Declaration of Helsinki and was approved by our ethics committee 『Approval No. 2020-20区. Patients were not required to give informed consent to the study because the analysis used anonymous clinical data that obtained after each patient agreed to receive treatment for acute cholangitis. For disclosure, the details of study are posted on some walls in National Hospital Organization Kure Medical Center and Chugoku Cancer Center.

\section{पExamination on admission}

All patients were checked for vital signs and underwent abdominal computed tomography scans. Blood samples were obtained on admission. Blood culture was assessed and a differential WBC count, including Neut\%, CRP, PCT, and other laboratory data, was undertaken. The hematology tests were performed with a Sysmex Hematology XN Modular System (Sysmex Co. Ltd., Hyogo, Japan) [10].

\section{QDiagnosis of acute cholangitis, severity assessment, and indication for urgent or early biliary drainage}

TG13 was used for diagnosis and severity grading of acute cholangitis (Supplementary Tables 1, 2). Acute cholangitis was defined using suspected or definite criteria. Severity criteria involved organ dysfunction (hypotension, disturbance of consciousness, respiratory dysfunction, oliguria or serum creatinine $>2.0 \mathrm{mg} / \mathrm{dl}$, prothrombin time international normalized ratio $>1.5$, and platelet count $<100,000 / \mathrm{mm}^{3}$ ). Moderate cholangitis-considered to pose a risk for severe cholangitis without early biliary drainage-was defined in the presence of more than two of the following five items: abnormal WBC count $\left(>12,000 / \mathrm{mm}^{3}\right.$ or $\left.<4,000 / \mathrm{mm}^{3}\right)$, high fever $\left(\geq 39^{\circ} \mathrm{C}\right)$, age $\geq 75$ years, hyperbilirubinemia (Total bilirubin $\geq 5.0 \mathrm{mg} / \mathrm{dL}$ ), and hypoalbuminemia (Albumin $<$ STD $\times 0.7=2.92$ at our institute) (Supplementary Table 2). Blood cultures were obtained twice in one session at initial diagnosis and one or two positive results were defined as a positive hemoculture and the presence of skin-resident bacteria was treated as a negative hemoculture. We defined the timing of biliary decompression as 'urgent' within 6 hours of admission and 'early' within 24 hours. Fundamentally, we performed urgent biliary drainage for severe cholangitis and early biliary drainage for moderate cholangitis using an endoscopic retrograde cholangiopancreatography technique.

\section{पAnalysis of characteristics of patients with positive and negative hemoculture}

We divided 94 patients into a positive hemoculture group and negative hemoculture group and compared the Neut\%, WBC, CRP, PCT, and other characteristics.

\section{UROC curve analysis}


Receiver operating characteristic (ROC) curve analysis was performed to evaluate the ability of Neut\%, WBC, CRP, and PCT to predict the presence of bacteremia and level of severity in patients with acute cholangitis.

\section{पPredictor for bacteremia in acute cholangitis}

We compared the ability of Neut\%, PCT, and other factors associated with the severity assessment criteria in TG13 to predict bacteremia.

\section{uPrognosis according to Neut\%}

We divided patients into higher Neut\% group and lower group and compared their prognosis.

\section{UUsefulness of Neut\% in mild acute cholangitis}

Finally, we divided patients with mild cholangitis into a positive hemoculture and negative hemoculture group and evaluated the ability of Neut\% to predict bacteremia in mild cholangitis.

\section{Statistical analysis}

A Fisher's exact test and $\chi^{2}$ test were used for categorical variables and the Welch $t$-test and Wilcoxon signed-rank test were used for quantitative data, where appropriate. A binomial regression analysis was performed to identify independent predictors of positive hemoculture. To evaluate the usefulness of Neut\%, WBC count, CRP, and PCT for prediction of positive hemoculture, ROC curves were plotted and areas under the curve (AUCs) were calculated with $95 \%$ confidence intervals $(\mathrm{Cl})$. Statistical evaluations of the AUC curve and the differences between each biomarker were performed using an $\chi^{2}$ test. The optimal cutoff values for Neut\% on the ROC curve were determined on the basis of the Youden index. A $P$ value of $<0.05$ was regarded as significant. The odds ratios were reported together with their $95 \%$ confidence limits. All statistical analyses of recorded data were performed using the Excel statistical software package (Ekuseru-Toukei 2015 version; Social Survey Research Information Co., Ltd., Tokyo, Japan).

\section{Results}

\section{uPatient characteristics}


Forty-eight patients out of 94 had a positive hemoculture and 46 were negative (Fig. 1). The prevalence of species in a positive hemoculture and patient characteristics are shown in Table 1, 2, respectively. Mean age, body temperature, and heart rate were significantly higher in patients with a positive hemoculture than those with a negative hemoculture. Platelet count and serum amylase levels were also lower in patients with a positive hemoculture. The levels of inflammatory markers including as WBC, CRP, and PCT did not differ significantly in the two groups. Neut\% was significantly higher in the positive hemoculture group $(91.7 \pm 4.0 \%$ vs. $82.5 \pm 9.0 \%, P<0.0001)$. In the multivariate analysis (Odds ratio(OR) 1.52 [1.16-1.15], $P=0.0030$ ), Neut\% was identified as the only factor that differed significantly in the two groups. Patients diagnosed with a positive hemoculture were more likely to have moderate or severe cholangitis than those with a negative hemoculture $(35 / 48,72.9 \%$ vs. $17 / 46,37 \%, P=0.0004)$. Urgent or early biliary drainage was performed in more patients with positive hemoculture than with negative hemoculture $(31 / 48,64.6 \%$ vs. $17 / 46,37 \%$, $P=0.0065)$. Bacteremia and subsequent high Neut\% were considered to be associated with patient prognosis.

Table 1. Results of blood cultures in patients with acute cholangitis

\begin{tabular}{|c|c|}
\hline Species & $\begin{array}{l}\text { Positive } \\
\text { Hemoculture } \\
(\mathrm{N}=48)\end{array}$ \\
\hline Escherichia coli & 22 \\
\hline Escherichia coli (ESBL) & 2 \\
\hline Escherichia coli + Enterococcus avium & 1 \\
\hline Escherichia coli + Pseudomonas aeruginosa & 1 \\
\hline Klebsiella pneumoniae & 9 \\
\hline Klebsiella pneumoniae + Enterococcus casseliflavus & 1 \\
\hline Klebsiella pneumoniae + Streptococcus anginosus & 1 \\
\hline Klebsiella Oxytoca & 1 \\
\hline Klebsiella spp & 1 \\
\hline Pseudomonas aeruginosa & 3 \\
\hline Citrobacter SPP & 1 \\
\hline Citorobacter braakii & 1 \\
\hline Enterococcus casseliflavus & 2 \\
\hline Streptococcus anginosus + Enterobacter aerogenes & 1 \\
\hline Clostridium perfringens & 1 \\
\hline
\end{tabular}

Table 2. Characteristics at the time of initial diagnosis and outocomes according to the result of hemoculture 


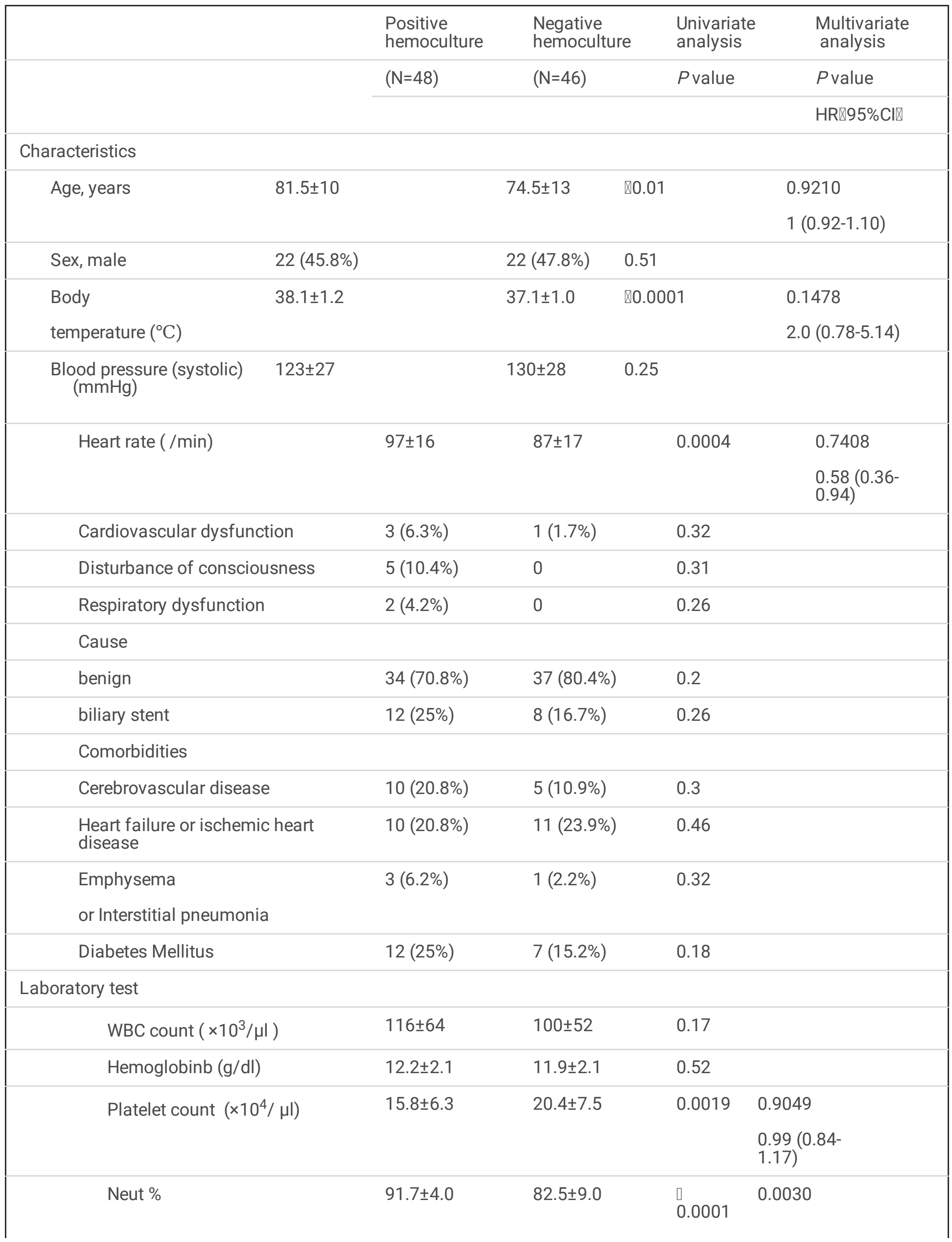




\begin{tabular}{|c|c|c|c|}
\hline Lymph \% & $5.0 \pm 2.9$ & $11.3 \pm 7.3$ & $\varangle 0.0001$ \\
\hline Mono \% & $2.7 \pm 1.8$ & $4.9 \pm 2.7$ & $\nabla 0.0001$ \\
\hline Albumin (g/dl) & $3.5 \pm 0.6$ & $3.7 \pm 0.6$ & 0.045 \\
\hline
\end{tabular}

\subsection{2 \\ $1.00(0.20-$ \\ 5.09)}

AST (IU/I)
ALT (IU/I)
ALP (IU/I)

Total bilirubin (mg/dl)

Amylase (IU/I)

$283 \pm 276$

$197 \pm 196$

$908 \pm 551$

$2.8 \pm 1.6$

$70 \pm 42$

196

$235 \pm 286$

$196 \pm 271$

$929 \pm 646$

$4.3 \pm 5.9$

$101 \pm 83$

0.41

0.98

0.87

0.08

0.026

0.7754

1.00 (0.99-

1.02)

Blood urea nitrogen $(\mathrm{mg} / \mathrm{dl})$

$28 \pm 25$

$20 \pm 11$

0.054

0.8302

1.02 (0.86-

121)

Creatinine $(\mathrm{mg} / \mathrm{dl})$

$1.23 \pm 1.09$

$0.95 \pm 0.48$

0.11

0.2057

23.5 (0.18-

3106.26)

$\mathrm{CRP}(\mathrm{mg} / \mathrm{dl})$
Procalcitonin $₫ \mathrm{ng} / \mathrm{ml} \rrbracket$

\section{PT- INR}

Severity of cholangitis

mild : moderate : severe

moderate + severe

severe

$7.0 \pm 7.9$

$21.5 \pm 50.4$

$1.21 \pm 0.35$

$13 \llbracket 22 \rrbracket 13$

$35(72.9 \%)$

$29 \llbracket 12 \rrbracket 5$

$17(37 \%)$

$5.9 \pm 5.7$
$4.3 \pm 9.1$

$1.20 \pm 0.28$

0.96

0.43

0.055

$13(27 \%)$

$5(10.9 \%)$

0.12

0.0019

0.0004

0.8814

0.82 (0.07-

10.41)

0.1745

12.9 (0.32-

514.9)

Outcome

\begin{tabular}{llll}
$\begin{array}{l}\text { Duration of anitibiotics usage } \\
\text { (day) }\end{array}$ & $8.1 \pm 5.0$ & $7.1 \pm 4.0$ & 0.3 \\
\hline Urgent /early biliary drainage & $\begin{array}{l}31 \\
(64.6 \%)\end{array}$ & $17(37 \%)$ & 0.0065 \\
\hline Death with cholangitis & $2(4.2 \%)$ & 0 & 0.43
\end{tabular}


The data of procalcitonin were obtained from 35 patients with positive hemoculture and 34 patients with negative hemoculture. Data are presented as number of patients (\%). Mean values are presented as mean \pm SD.

WBC=white blood cells; Neut\%=proportion of neutrophils in the white blood cells; Lymph\%= proportion of lymphocytes in the white blood cells; Mono\%=proportion of monocytes in the white blood cells; GOT=glutamic oxaloacetic transaminase; GPT= glutamic pyruvic transaminase; $\mathrm{CRP}=\mathrm{C}$-reactive protein; $\mathrm{PT}$-INR=prothrombin time international normalized ratio

\section{पAbility of Neut\% to predict bacteremia and severity in acute cholangitis}

Fig. 2 illustrates the ROC curves for Neut\%, WBC, CRP, and PCT for prediction of bacteremia (Fig. 2a), severe or moderate cholangitis (Fig. 2b), and severe cholangitis (Fig. 2c). Neut\% and PCT were significantly associated with bacteremia (Neut\%; AUC 0.86, 95\%Cl: 0.77-0.95, $P<0.001$, PCT; AUC 0.63, 95\%Cl: $0.50-0.77, P=0.047$ ) and the correlation was stronger for Neut\% compared to PCT ( $P=0.002)$. The optimal cutoff value using the Youden index in the ROC curve analysis was Neut\% $89.7 \%$ and the sensitivity and specificity of this Neut\% cutoff value for diagnosis of bacteremia was $77 \%$ and $80 \%$, respectively. The associations between Neut\%, PCT, and CRP and moderate or severe cholangitis were statistically significant (Neut\%; AUC 0.75, 95\% Cl: 0.63-0.87, $P<0.001, \mathrm{PCT}$; AUC 0.70, 95\%Cl: 0.60-0.82, $P=.0023, \mathrm{CRP}$; AUC $0.67,95 \% \mathrm{Cl}: 0.54-0.80, P=0.0128)$, but there was no statistical difference between Neut\% and PCT $(P=0.50)$. Regarding the prediction of severe cholangitis, Neut\%, PCT, and CRP showed good statistical power (Neut\%; AUC 0.71, 95\% Cl 0.56-0.86, $P=0.0068$, PCT; AUC 0.75, 95\%Cl 0.60-0.89, $P<0.001, \mathrm{CRP} ; \mathrm{AUC} 0.70,95 \% \mathrm{Cl}: 0.53-0.88, P=0.0234$ ), but there were no differences in ability among the three markers.

\section{पComparison of ability to predict bacteremia with other factors}

We compared the ability of Neut\% for prediction of bacteremia with PCT and other factors associated with severity assessment of acute cholangitis in TG13. We set the cutoff value of Neut\% at $89.7 \%$ using the Youden index in the ROC curve. There were more patients with bacteremia in the Neut\% $\geq 89.7 \%$ patients than in the Neut $\%<89.7 \%$ patients in the univariate analysis $(37 / 48,77.1 \%$ vs. $9 / 46,19.6 \%, P<0.0001)$ and Neut $\% \geq 89.7 \%$ and body temperature were selected as independent predictors of bacteremia in the multivariate analysis (Table 3 ).

Table 3. Analysis to identify predictors of bacteremia 


\begin{tabular}{|c|c|c|c|c|}
\hline & $\begin{array}{l}\text { Positive } \\
\text { Hemoculture }\end{array}$ & $\begin{array}{l}\text { Negative } \\
\text { Hemoculture }\end{array}$ & $\begin{array}{l}\text { Univariate } \\
\text { analysis }\end{array}$ & $\begin{array}{l}\text { Multivariate } \\
\text { analysis }\end{array}$ \\
\hline & $(\mathrm{N}=48)$ & $(\mathrm{N}=46)$ & $P$ value & $P$ value \\
\hline & & & & $\mathrm{HR} \rrbracket 95 \% \mathrm{Cl} \rrbracket$ \\
\hline \multirow[t]{2}{*}{ Age $\geqq 75$ years old } & 35 (72.9\%) & $27(58.7 \%)$ & 0.045 & 0.5930 \\
\hline & & & & $1.4(0.38-5.29)$ \\
\hline \multirow{2}{*}{ 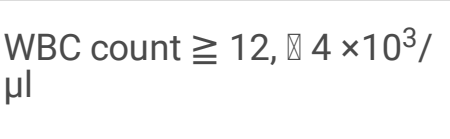 } & $25(52 \%)$ & $10(21.7 \%)$ & 0.0022 & 0.4834 \\
\hline & & & & $1.6(0.45-5.40)$ \\
\hline \multirow[t]{2}{*}{ Body temperature $\geqq 39^{\circ} \mathrm{C}$} & $14(29.2 \%)$ & $2(4.3 \%)$ & 0.0012 & 0.0101 \\
\hline & & & & $13.1(1.85-92.65)$ \\
\hline \multirow[t]{2}{*}{ Platelet count $\nabla 10 \times 10^{4} / \mu \mathrm{l}$} & $9(18.8 \%)$ & $1(2.2 \%)$ & 0.009 & 0.1154 \\
\hline & & & & $7.3(0.61-86.79)$ \\
\hline 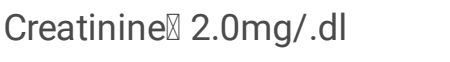 & $4(8.3 \%)$ & 0 & 0.064 & \\
\hline$P T-I N R \geqq 1.5$ & $3(6.3 \%)$ & $2(4.3 \%)$ & 0.52 & \\
\hline Albumin $\rrbracket 2.92 \mathrm{~g} / \mathrm{dl}$ & $13(27 \%)$ & $6(13 \%)$ & 0.075 & \\
\hline Total bilirubin $\geqq 5.0 \mathrm{mg} / \mathrm{dl}$ & $7(14.6 \%)$ & $11(23.9 \%)$ & 0.19 & \\
\hline \multirow[t]{2}{*}{ Neut $\% \geqq 89.7 \rrbracket$} & $39(81.3 \%)$ & $11(23.9 \%)$ & $\llbracket 0.0001$ & $\varangle 0.001$ \\
\hline & & & & $12.7(3.71-43.78)$ \\
\hline Procalcitonin $\geqq 2.0 \mathrm{ng} / \mathrm{ml}$ & $17(35.4 \%)$ & $11(23.9 \%)$ & 0.13 & \\
\hline Procalcitonin $\geqq 0.5 \mathrm{ng} / \mathrm{ml}$ & $26(54.1 \%)$ & $18(39.1 \%)$ & 0.055 & \\
\hline \multirow{2}{*}{$\begin{array}{l}\text { Severe or moderate } \\
\text { cholangitis }\end{array}$} & $35(72.9 \%)$ & $17(37.0 \%)$ & 0.00044 & 0.9990 \\
\hline & & & & $1.0(0.28-3.62)$ \\
\hline
\end{tabular}

The data of procalcitonin were obtained from 35 patients with positive hemoculture and 34 patients with negative hemoculture. Data are presented as number of patients (\%). Mean values are presented as mean \pm SD.

The data of procalcitonin were obtained from 35 patients with positive hemoculture and 34 patients with negative hemoculture. Data are presented as number of patients (\%). Mean values are presented as mean \pm SD.

WBC=white blood cells; PT-INR=prothrombin time international normalized ratio; Neut\%=proportion of neutrophils in the white blood cells

\section{uPrognosis according to Neut\%}

We divided 94 patients into a Neut\% $\geq 89.7 \%$ group $(n=46)$ and a Neut $\%<89.7 \%$ group $(n=48)$ and compared the prognosis in both groups (Table 4). In the Neut\% $\geq 89.7 \%$ group, there were more patients with moderate or severe cholangitis $(34 / 46(73.9 \%)$ vs. $20 / 48(37.5 \%), P=0.0015)$ and the frequency of urgent or early biliary decompression 
(within 24 hours) was higher than in the Neut\% $<89.7 \%$ group $(30 / 46,65.2 \%$ vs. $18 / 48,37.5 \%, P=0.0063)$. Furthermore, hospital days were longer in $\geq 89.7 \%$ group than in Neut\% $<89.7 \%$ group (median: 18 days vs. 14 days, $P=0.04$ ).

Table 4. Prognosis according to Neut\%

\begin{tabular}{|c|c|c|c|}
\hline & Neut $\% \geqq 89.7 \%$ & Neut\%\89.7\% & Univariate analysis \\
\hline & $(\mathrm{N}=46)$ & $(\mathrm{N}=48)$ & $P$ value \\
\hline Severity of cholangitis (severe) & $13(28.3 \%)$ & $6(12.5 \%)$ & 0.049 \\
\hline Severity of cholangitis (moderate or severe) & $34(73.9 \%)$ & $20(41.7 \%)$ & 0.0015 \\
\hline Urgent or early biliary drainage & $30(65.2 \%)$ & $18(37.5 \%)$ & 0.0063 \\
\hline Duration of antibiotics (day) & 7 (3-33) & $7(2-20)$ & 0.96 \\
\hline Hospital stay (day) & $18(7-56)$ & $14(3-119)$ & 0.04 \\
\hline Death associated with cholangitis & $2(4.3 \%)$ & 0 & 0.24 \\
\hline
\end{tabular}

Data except duration of antibiotics and hospital days are presented as number of patients (\%).

Duration of antibiotics and hospital days are presented as median values.

\section{$\square$ Ability of Neut\% to predict bacteremia in mild cholangitis}

In patients with mild cholangitis according to TG13, close observation without biliary drainage is recommended. However, 13 of 40 patients with mild cholangitis had a positive hemoculture in this study. As such, we studied the features of patients who had bacteremia even if they were diagnosed with mild cholangitis. Table $\mathbf{5}$ shows the characteristics of patients with mild cholangitis. There were significant differences between patients with positive and negative hemoculture in Neut\% and platelet count in the univariate analysis (Neut\%: $89.4 \% \pm 4.4 \%$ vs. $81.8 \% \pm 8.7 \%$, $P=0.0007$, platelet count: $17.1 \pm 3.6 \times 10^{4}$ vs. $21.7 \pm 7.4 \times 10^{4} / \mu \otimes, P=0.013$ ) and in Neut\% in the multivariate analysis $(P=0.046)$. We compared the ability of Neut\% for prediction of bacteremia with other factors associated with severity assessment of acute cholangitis in TG13. We set the platelet count value at $<19.4 \times 10^{4} / \mu \mathrm{L}$ using the mean value of the positive hemoculture group and the negative hemoculture group, and compared the ability of Neut\% for prediction of bacteremia with other factors (Table 6). In the univariate analysis, there were significant differences in Neut $\% \geq 89.7 \%$ and platelet count $<19.4 \times 10^{4} / \mu \mathrm{L}$ (Neut\%: $9 / 13,69.2 \%$ vs. $4 / 27,14.8 \%, P=0.0097$, platelet count $\times 10^{4} / \mu \mathrm{L}: 11 / 13,84.6 \%$, $13 / 27,48.1 \%, P=0.029)$. In the multivariate analysis, Neut $\% \geq 89.7 \%$ was the only independent factor associated with bacteremia in mild cholangitis $(P=0.016)$.

Table 5. Characteristics of the patients with mild cholangitis 


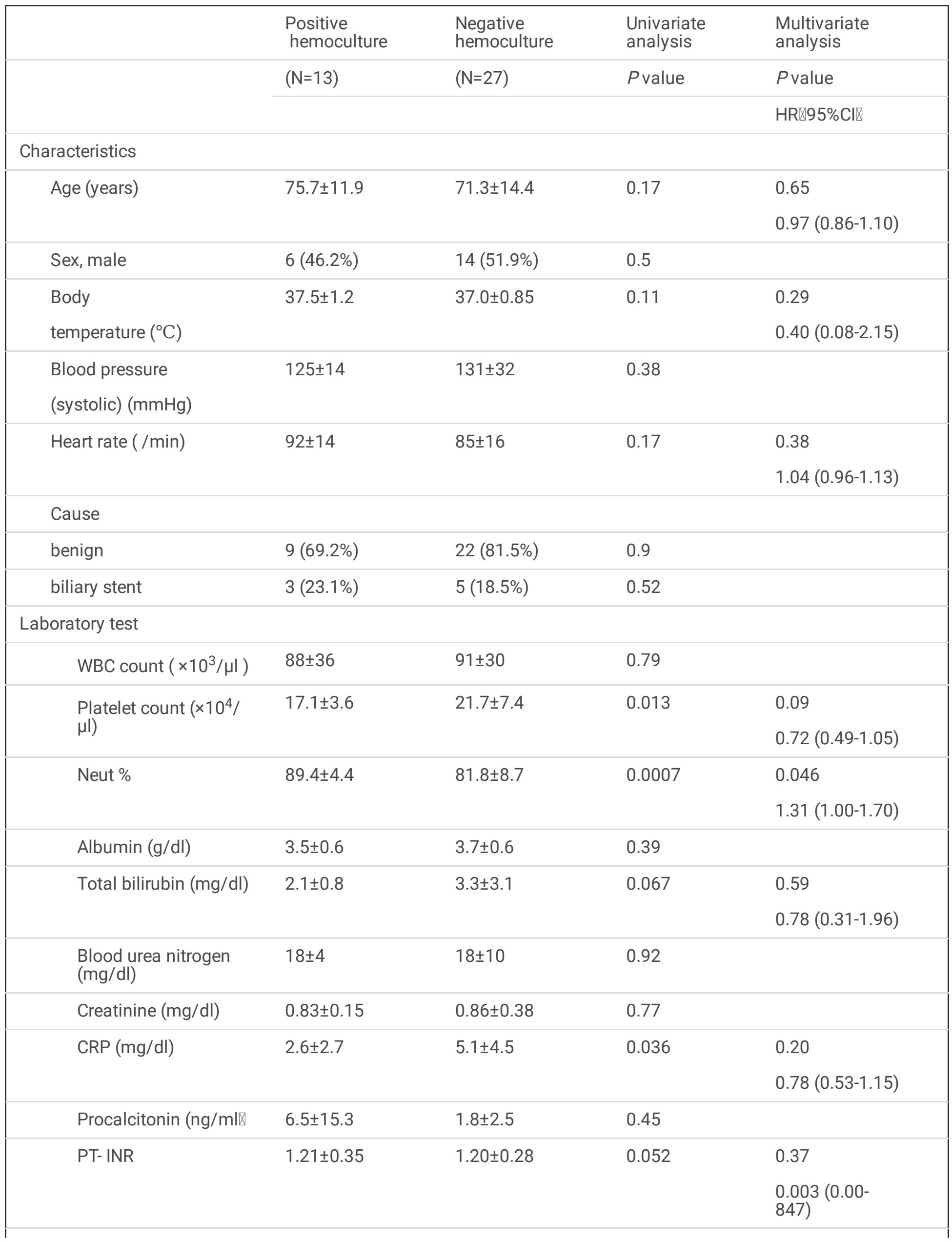


The data of procalcitonin were obtained from 7 patients with positive hemoculture and 21 patients with negative hemoculture. Data are presented as number of patients (\%). Mean values are presented as mean \pm SD.

WBC=white blood cells; Neut\%=proportion of neutrophils in the white blood cells; CRP=C-reactive protein; PTINR=prothrombin time international normalized ratio

Table 6. Analysis to identify predictors of bacteremia in patients with mild cholangitis

\begin{tabular}{|c|c|c|c|c|}
\hline & $\begin{array}{l}\text { Positive } \\
\text { Hemoculture }\end{array}$ & $\begin{array}{l}\text { Negative } \\
\text { Hemoculture }\end{array}$ & $\begin{array}{l}\text { Univariate } \\
\text { analysis }\end{array}$ & $\begin{array}{l}\text { Multivariate } \\
\text { analysis }\end{array}$ \\
\hline & $(\mathrm{N}=13)$ & $(\mathrm{N}=27)$ & $P$ value & $P$ value \\
\hline & & & & HR $₫ 95 \% \mathrm{CI} \rrbracket$ \\
\hline \multirow[t]{2}{*}{ Sex, male } & $6(46.2 \%)$ & $14(51.9 \%)$ & 0.5 & 0.289 \\
\hline & & & & $0.33(0.04-2.58)$ \\
\hline \multirow[t]{2}{*}{$\mathrm{Age} \geqq 75$ years old } & $7(53.8 \%)$ & $27(48.1 \%)$ & 0.5 & 0.117 \\
\hline & & & & $1.04(0.99-1.08)$ \\
\hline$W B C \geqq 120, \nabla 40 \times 10^{3} / \mu \mathrm{l}$ & $4(30.8 \%)$ & $3(11.1 \%)$ & 0.14 & \\
\hline Body temperature $\geqq 39^{\circ} \mathrm{C}$ & $7(53.8 \%)$ & $13(48.1 \%)$ & 0.5 & \\
\hline \multirow{2}{*}{ 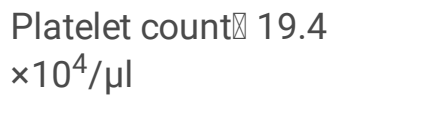 } & $11(84.6 \%)$ & $13(48.1 \%)$ & 0.029 & 0.259 \\
\hline & & & & $3.67(0.38-34.98)$ \\
\hline Albumin $\nabla 2.92 \mathrm{~g} / \mathrm{dl}$ & $1(7.6 \%)$ & $2(7.4 \%)$ & 0.7 & \\
\hline $\begin{array}{l}\text { Total bilirubin } \geqq 5.0 \\
\mathrm{mg} / \mathrm{dl}\end{array}$ & 0 & $4(14.8 \%)$ & 0.19 & \\
\hline $\begin{array}{l}\mathrm{CRP}(\mathrm{mg} / \mathrm{dl}) \geqq 3.85 \\
\mathrm{mg} / \mathrm{dl}\end{array}$ & $5(38.5 \%)$ & $8(51.9 \%)$ & 0.33 & \\
\hline \multirow[t]{2}{*}{ Neut $\% \geqq 89.7 \rrbracket$} & $8(61.5 \%)$ & $5(18.5 \%)$ & 0.0097 & 0.016 \\
\hline & & & & $9.85(1.53-63.19)$ \\
\hline Procalcitonin $\geqq 2.0 \mathrm{ng} / \mathrm{ml}$ & $2(28.6 \%)$ & $7(25.9 \%)$ & 0.6 & \\
\hline Procalcitonin $\geqq 0.5 \mathrm{ng} / \mathrm{ml}$ & $3(23.1 \%)$ & $10(47.6 \%)$ & 0.59 & \\
\hline
\end{tabular}

The data of procalcitonin were obtained from 7 patients with positive hemoculture and 21 patients with negative hemoculture. Data are presented as number of patients (\%). Mean values are presented as mean \pm SD.

$\mathrm{WBC}=$ white blood cells; $\mathrm{CRP}=\mathrm{C}$-reactive protein; Neut\%=proportion of neutrophils in the white blood cells

\section{Discussion}

To the best of our knowledge, this study is the first to show the usefulness of Neut\% for prediction of bacteremia in patients with focusing on only acute cholangitis. Neut\% showed an excellent independent diagnostic ability for 
bacteremia in the ROC curve analysis (AUC in ROC analysis was 0.86 , and diagnostic sensitivity was $77 \%$ and specificity was $80 \%$ for Neut $\% \mathbf{2} 8.7 \%$ ), and there were more patients with moderate and severe cholangitis and more patients requiring urgent or early biliary drainages in the high Neut\% group than in the low Neut\% group. Based on these results, Neut\% was thought to be an excellent predictor, not only for bacteremia, but also for prognosis of cholangitis.

Furthermore, measurement of Neut\% is easy, quick, and inexpensive. Neut\% may be useful for care of patients with acute cholangitis, especially in terms of the decision to perform early biliary drainage.

Acute cholangitis can be fatal and urgent or early biliary decompression is required according to severity. Factors associated with prognosis (mortality and/or need for early biliary decompression) have been reported as WBC count, hyperbilirubinemia, hypoalbuminemia, acute renal failure, shock, thrombocytopenia, endotoxemia, high fever, comorbidities, advanced age, malignant disease, extension of prolonged prothrombin time, smoking, and bacteremia $[2,8,11-15]$. Some researchers have developed scoring systems using various prognostic factors with good results $[2,12,16,17]$. TG13 [9] also assesses severity using several prognostic factors. It is an easy and effective scoring system, its usefulness has been confirmed, and it was taken over to the Updated Tokyo Guidelines for acute cholangitis and cholecystitis in 2018 [18,19]. Furthermore, PCT [6-8], endotoxin activity [20,21], NF-kB [22], IL-7 [23], delta-neutrophil index [24], and presepsin [25] have been reported as independent biomarkers for prognosis of acute cholangitis and PCT [6,23], endotoxin activity [20], and IL-7 [23] for bacteremia.

In our clinical practice, we have observed that patients with bacteremia and/or severe cholangitis often have a high Neut\% value (for example, Neut\% $\geq 90 \%$ ). Nowadays, at any hospital in Japan, neutrophil levels, lymphocytes, eosinophil levels, and monocytes among WBCs (Neut\%, Lymp\%, Eosi\%, and Mono\%) are measured automatically and quickly in an easy method, and the results are extremely accurate [10]. In bacterial and fungal infections, the number of mature neutrophils increases in the blood, and the neutrophil level (the amount of mature and young neutrophils), Neut\%, and WBC count are up-regulated [26,27]. If the inflammation becomes severe as in sepsis, the younger neutrophils are released into the peripheral blood and there is a neutrophil left shift. WBC count and neutrophil count are thought to be the earliest and the most sensitive inflammatory markers and can be used to evaluate the treatment effect $[26,27]$. Therefore, we studied the association between bacteremia and Neut\%, and found that Neut\% was excellent for diagnosis of a positive hemoculture. Some studies have reported that Neut\% is a useful marker for bacteremia in infections. Fukui et al. [28] stated that a Neut\% $>80 \%$ was an independent risk factor for a positive hemoculture. In this time, we studied correlation between bacteremia and Neut\% focusing on only acute cholangitis. Further, one report [29] concluded that a high neutrophil-to-lymphocyte ratio (NLR) was a very good independent predictor of lethal outcomes in critically ill patients with secondary sepsis and/or trauma. We presume that NLR is nearly equal to Neut\%. The neutrophil left shift is used for diagnosis of sepsis and it is a possible predictive factor for bacteremia. Kim et al. reported the efficacy of measuring the delta neutrophil index (DNI) as a parameter of neutrophil left shift for prediction of prognosis in patients with acute cholangitis [24]. However, it is difficult to obtain results for the DNI and it is difficult to obtain a blood smear for all patients. In accordance with results of this study, the up-regulation of Neut\% may show the increasing necessity of neutrophil and neutrophil left shift assessments.

WBC count is the most common inflammatory biomarker, and many authors have reported the usefulness of WBC count as a predictor of severe acute cholangitis. However, our study and some other authors [6,7] concluded that WBC count was not independently useful for prognosis and bacteremia in acute cholangitis. A reason for this may be that the reaction of the WBC count in response to bacterial or fungal infections differs in each patient, especially in the elderly, and another reason is that the WBC count may change from high to low over the course of treatment of a severe case, and in severe inflammation.

Many authors have suggested that serum PCT level is a superior predictor of the presence of severe acute inflammation compared to conventional markers such as WBC count and serum CRP levels [30]. In this study, PCT showed a good and

Page $13 / 18$ 
equal ability for assessment of severity compared with Neut\% in the ROC curve analysis, but the diagnostic ability for bacteremia was weaker than Neut\%. In general, in most diseases, bacteremia occurs with extensive and prolonged inflammation, but bacteremia in acute cholangitis occurs early and easily with the onset of obstruction of the bile duct because the cause of bacteremia is cholangio-venous reflux by up-regulation of pressure in the bile duct. Therefore, in acute cholangitis, it is important to identify infection in the early phase after onset. Although PCT is readily produced within 6-12 h, and serum procalcitonin levels increase [30], we presume that the earliest biomarker for bacteremic cholangitis is not PCT, CRP, or WBC-it is Neut\%.

The recommendation for patients with mild cholangitis is observation without biliary drainage in TG13/18; however, we had 13 bacteremic patients of 40 patients with mild cholangitis. These patients may have needed early biliary decompression after considering other examination results. In this study, Neut\% was an excellent predictor of bacteremia in not only all patients with cholangitis, but also patients with mild cholangitis. Accordingly, high Neut\% can be utilized as an indicator for early biliary decompression in patients with mild cholangitis in TG13/18.

This study had several limitations. First, this was a retrospective analysis of medical records at a single hospital. There were some patients with acute cholangitis who did not have a blood culture test and there were no criteria on which to base the decision to obtain a blood culture or not. Patients who underwent a blood culture test had acute cholangitis with suspicion of bacteremia and severe cholangitis, and as such, our analysis using this population was relatively reasonable. Second, there was some data deficiency in serum PCT levels; therefore, this analysis may not have provided a completely correct assessment of the association between Neut\% and PCT. Third, in this study, patients with acute pancreatitis and usage of an anticancer drug were excluded because these conditions would affect WBC count, Neut\%, and CRP. In acute pancreatitis, not only the severity assessment, but also the diagnosis of cholangitis is difficult, and Neut\% is relatively high in acute pancreatitis. Thus, predicting bacteremia in patients with acute pancreatitis is challenging. The anticancer drugs for pancreato-biliary cancer, gemcitabine, S-1, nab-paclitaxel, and cisplatin, do not induce severe bone marrow suppression compared with drugs for other cancers, and as such, there is often a high Neut\% with a normal or low WBC count in patients with bacteremic acute cholangitis (data not shown). We think that high Neut\% is an important factor for consideration when bacteremia is suspected in a patient taking an anticancer drug.

\section{Conclusions}

In conclusion, the percentage of neutrophils in the WBC count is measured automatically with an easy and quick method and might be useful for predicting bacteremia and severity of acute cholangitis during initial medical examination. If the Neut\% is high, the existence of bacteremia should be considered, and early biliary drainage should be performed.

\section{Declarations}

\section{पEthics approval and consent participate}

This study was performed in accordance with the Declaration of Helsinki and was approved by our ethics committee \Approval No. 2020-20区. Patients were not required to give informed consent to the study because the analysis used anonymous clinical data that obtained after each patient agreed to receive treatment for acute cholangitis. For disclosure, the details of study are posted on some walls in National Hospital Organization Kure Medical Center and Chugoku Cancer Center.

\section{口Consent for publication}


Patients were not required to give informed consent to the study because the analysis used anonymous clinical data that obtained after each patient agreed to receive treatment for acute cholangitis. For disclosure, the details of study are posted on some walls in National Hospital Organization Kure Medical Center and Chugoku Cancer Center.

\section{पAvailability of data and materials}

The datasets used and/or analysed during the current study are available from the corresponding author on reasonable request.

\section{पCompeting interests}

The authors declare that they have no competing interests.

\section{DFunding}

No funding was received.

\section{¿Author's contribution}

Conceptualization: AY

Writing: AY

Acquisition of patients' data: KW, RM, KT, HK1 corresponding to Hirona Konishi, KI, NH, TS

Writing-review: YT, RK, TK, HK2 corresponding to Hirotaka Kouno, HK3 corresponding to Hiroshi Kohno

All authors have read and approved the manuscript.

पAcknowledgment We thank all clinical technologists and our colleagues at the Kure Medical Center and Chugoku Cancer Center. The authors wish to thank Kanami Okino for helping collect the data and conduct the literature search.

\section{Abbreviations}

PCT: serum procalcitonin, Neut\%: the neutrophil proportion in the white blood cell count, TG13: the updated Tokyo guidelines for acute cholangitis and acute cholecystitis 2013, WBC: white blood cell count, CRP: C-reactive protein, ROC: receiver operating characteristic, AUC: area under the curve, OR: odds ratio, Cl: confidence interval, NLR: neutrophil-tolymphocyte ratio, DNI: the delta neutrophil index

\section{References}


1. Tan M, Schaffalitzky de Muckadell OB, Laursen SB. Association Between Early ERCP and Mortality in Patients With Acute Cholangitis. Gastrointest Endosc 2018;87:185-92.

2. Tompson J, Beninion RS, Pitt HA. An analysis of infectious failures in acute cholangitis. HPB Surg 1994;8:139-44.

3. Gigot JF, Leese T, Dereme T, Coutinho J, Castaing D, Bismuth H. Acute cholangitis. Multivariate analysis of risk factors. Ann Surg 1989;209:435-8.

4. Lee CC, Chang IJ, Lai YC, Chen SY, Chen SC. Epidemiology and prognostic determinants of patients with bacteremic cholecystitis or cholangitis. Am J Gastroenterol 2007;102:563-9.

5. Lee BS, Hwang JH, Lee SH, Jang SE, Jang ES, Jo HJ, et al. Risk factors of organ failure in patients with bacteremic cholangitis. Dig Dis Sci 2013;58:1091-9.

6. Shinya S, Sasaki T, Yamashita Y, Kato D, Yamashita K, Nakashima R, et al. Procalcitonin as a useful biomarker for determining the need to perform emergency biliary drainage in cases of acute cholangitis. J Hepatobiliary Pancreat Sci 2014;21:777-85.

7. Umefune G, Kogure H, Hamada T, Isayama H, Ishigaki K, Takagi K, et al. Procalcitonin Is a Useful Biomarker to Predict Severe Acute Cholangitis: A Single-Center Prospective Study. J Gastroenterol 2017;52:734-45.

8. Lee YS, Cho KB, Park KS, Lee JY, Lee YJ. Procalcitonin as a Decision-Supporting Marker of Urgent Biliary Decompression in Acute Cholangitis. Dig Dis Sci 2018;63:2474-9.

9. Kiriyama S, Takada T, Strasberg SM, Solomkin JS, Mayumi T, Pitt HA, et al. TG13 guidelines for diagnosis and severity grading of acute cholangitis (with video). J hepatobiliary Pancreat Sci 2013;20:24-34.

10. Briggs C, Longair I, Kumar P, Singh D, Machin SM. Performance evaluation of the Sysmex haematology XN modular system. J Clin Pathol 2012;65:1024-30.

11. Csendes A, Diaz JC, Burdiles P, Maluenda F, Morales E. Risk Factors Classification of Acute Suppurative Cholangitis. Br J Surg 1992;79:655-8.

12. Hui CK, Lai KC, Yuen MF, Ng M, Lai CL, Lam SK. Acute cholangitis-predictive factors for emergency ERCP. Aliment Pharmacol Ther 2001;15:1633-7.

13. Hanau LH, Steigbigel NH. Acute (ascending) cholangitis. Infect Dis Clin North Am 2000;14:521-46. Review.

14. Pang YY, Chun YA. Predictors for emergency biliary decompression in acute cholangitis. Eur J Gastroenterol Hepatol 2006;18:727-31.

15. Rosing DK, De Virgilio C, Nguyen AT, El Masry M, Kaji AH, Stabile BE. Cholangitis: analysis of admission prognostic indicators and outcomes. Am Surg 2007;73: 949-54.

16. Salek J, Livote E, Sideridis K, Bank S. Analysis of risk factors predictive of early mortality and urgent ERCP in acute cholangitis. J Clin Gastroenterol 2009;43:171-5.

17. Schneider J, Hapfelmeier A, Thöres S, Obermeier A, Schulz C, Pförringer D, et al. Mortality Risk for Acute Cholangitis (MAC): a risk prediction model for in-hospital mortality in patients with acute cholangitis. BMC Gastroenterol 2016;16:15. 18. Nishino T, Hamano T, Mitsunaga Y, Shirato I, Shirato M, Tagata T, et al. Clinical evaluation of the Tokyo Guidelines 2013 for severity assessment of acute cholangitis. J Hepatobiliary Pancreat Sci 2014;21:841-9.

19. Kiriyama S, Kozaka K, Takada T, Strasberg SM, Pitt HA, Gabata T, et al. Tokyo Guidelines 2018: Diagnostic Criteria and Severity Grading of Acute Cholangitis (With Videos). J Hepatobiliary Pancreat Sci 2018;25:17-30.

20. Sato M, Matsuyama R, Kadokura T, Mori R, Kumamoto T, Nojiri K, et al. Severity and prognostic assessment of the endotoxin activity assay in biliary tract infection. J Hepatobiliary Pancreat Sci 2014;21:120-7.

21. Lau JY, Chung SC, Leung JW, Ling TK, Yung MY, Li AK. Endoscopic drainage absorts endotoxaemia in acute cholangitis. Br J Surg 1996;83:181-4.

22. Gong JP, Liu CA, Wu CX, Wu CX, Li SW, Shi YJ, et al. Nuclear factor kB activity in patients with acute cholangitis. World J Gastroenterol 2002;8:346-9.

23. Suwa Y, Matsuyama R, Goto K, Kadokura T, Sato M, Mori R, et al. IL-7 and Procalcitonin Are Useful Biomarkers in the Comprehensive Evaluation of the Severity of Acute Cholangitis. J Hepatobiliary Pancreat Sci 2017;24:81-8. 
24. Kim H, Kong T, Chung SP, Hong JH, Lee JW, Joo Y, et al. Usefulness of the Delta Neutrophil Index as a Promising Prognostic Marker of Acute Cholangitis in Emergency Departments. Shock 2017;47:303-12.

25. Lin J, Sun H, Li J, Zheng Y, Shao C, Zhang YH, et al. Role of Presepsin for the Assessment of Acute Cholangitis Severity. Clin Lab 2016;62:679-87.

26. Cartwright GE, Athens JW, Wintrobe MM. THE KINETICS OF GRANULOPOIESIS IN NORMAL MAN. Blood 1964;24:780-803.

27. Honda T, Uehara T, Matsumoto G, Arai S, Sugano M. Neutrophil left shift and white blood cell count as markers of bacterial infection. Clin Chim Acta 2016;457:46-53.

28. Fukui S, Uehara Y, Fujibayashi K, Takahashi O, Hisaoka T, Naito T. Bacteremia predictive factors among general medical inpatients: a retrospective cross-sectional survey in a Japanese university hospital. BMJ Open 2016;6:e101527. 29. Djordjevic D, Rondovic G, Surbatovic M, Stanojevic I, Udovicic I, Andjelic T, et al. Neutrophil-to-Lymphocyte Ratio, Monocyte-to-Lymphocyte Ratio, Platelet-to-Lymphocyte Ratio, and Mean Platelet Volume-to-Platelet Count Tation as Biomarkers in Critically III and Injured Patients: Which Ration to Choose to Predict Outcome and Nature of Bacteremia. Mediators of inflammation 2018;2018:3758068.

30. Liu D, Su LX, Han G, Yan P, Xie L. Prognostic Value of Procalcitonin in Adult Patients with Sepsis: A Systematic Review and Meta-Analysis. PLoS One 2015;10:e0129450.

\section{Figures}

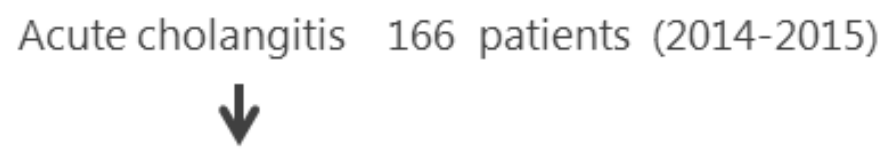

Had a blood culture without

pre-administration of antibiotics 115

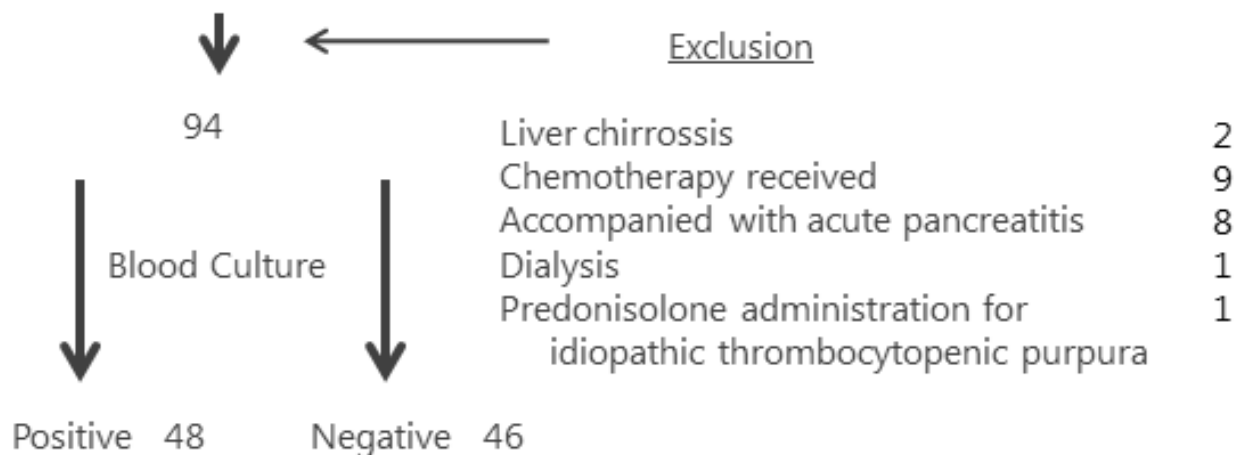

\section{Figure 1}

Flowchart for inclusion of patients with acute cholangitis The diagnosis of acute cholangitis is determined based on the Updated Tokyo Guidelines 2013 for severity evaluation of acute cholangitis. Patients with factors affecting white blood cell (WBC) count, neutrophil percentage on the WBC count, C-reactive protein, and body temperature are excluded. 
a ROC curve for bacteremia

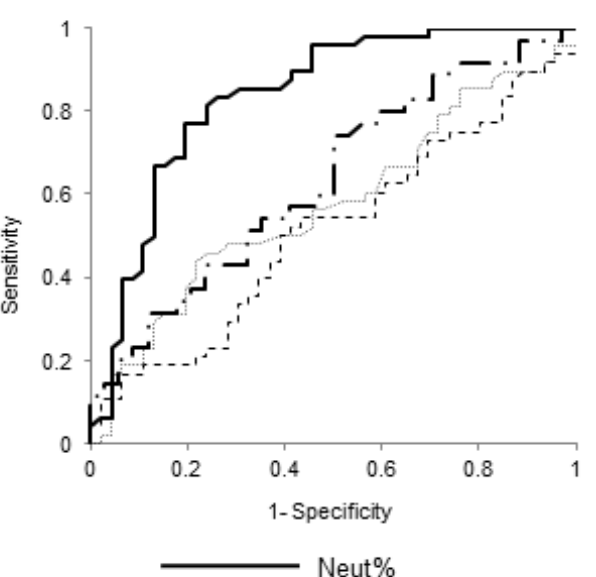

\begin{tabular}{lccc}
\hline & AUC & $95 \% \mathrm{Cl}$ & $\mathrm{P}$ \\
\hline Neut $\%$ & 0.86 & $0.77-0.95$ & $<0.001$ \\
PCT & 0.63 & $0.50-0.77$ & 0.047 \\
CRP & 0.51 & $0.37-0.64$ & 0.939 \\
WBC & 0.59 & $0.45-0.72$ & 0.221 \\
& & & \\
Neut $\%$ & vs. & PCT & 0.002 \\
Neut $\%$ & vs. & CRP & $<0.001$ \\
Neut $\%$ & vs. & WBC & $<0.001$
\end{tabular}

b ROC curve for moderate or severe cholangitis
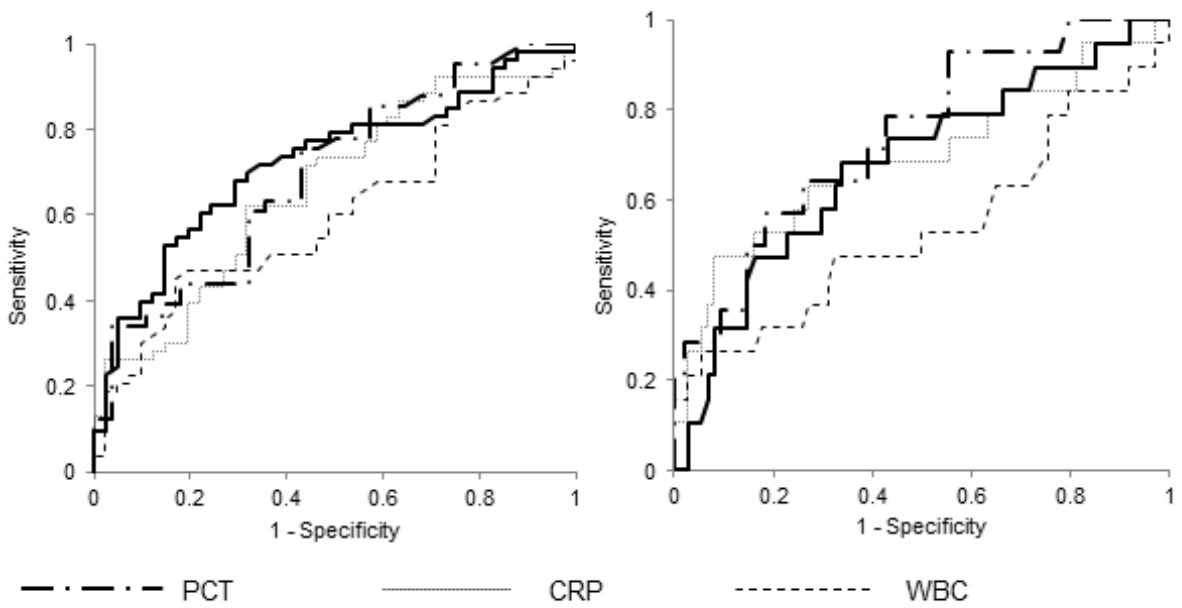

\begin{tabular}{lccc}
\hline & AUC & $95 \% \mathrm{Cl}$ & $\mathrm{P}$ \\
\hline Neut $\%$ & 0.75 & $0.63-0.87$ & $<0.001$ \\
PCT & 0.7 & $0.60-0.82$ & 0.0023 \\
CRP & 0.67 & $0.54-0.80$ & 0.012 \\
WBC & 0.59 & $0.46-0.73$ & 0.1763
\end{tabular}

\begin{tabular}{lccc}
\hline & AUC & $95 \% \mathrm{Cl}$ & $\mathrm{P}$ \\
\hline Neut $\%$ & 0.71 & $0.56-0.86$ & 0.0068 \\
PCT & 0.75 & $0.60-0.89$ & $\mathrm{P}<0.001$ \\
CRP & 0.7 & $0.53-0.88$ & 0.0234 \\
WBC & 0.51 & $0.31-0.71$ & 0.908 \\
& & & \\
Neut $\%$ & vs. & PCT & 0.699 \\
Neut $\%$ & vs. & CRP & 0.964 \\
Neut $\%$ & vs. & WBC & 0.209
\end{tabular}

Figure 2

The receiver operator characteristic (ROC) curve for proportion of Neutrophils in the white blood cells (Neut\%), white blood cells (WBC), C-reactive protein (CRP), and procalcitonin (PCT) for prediction of bacteremia, moderate or sevre cholangitis, and severe cholangitis. (a) Bacteremic versus non-Bacteremic acute cholagitis. (b) Moderate/severe versus mild acute cholangitis. (c) Severe versus mild/moderate acute cholangitis. AUC area under the curve, $\mathrm{Cl}$ confidence interval

\section{Supplementary Files}

This is a list of supplementary files associated with this preprint. Click to download.

- SupplementTable1.docx

- SupplementalTable2.docx 